\title{
Gene therapy for human colorectal cancer cell lines with recombinant adenovirus 5 based on loss of the insulin-like growth factor $\mathbf{2}$ imprinting
}

\author{
HUILING SUN ${ }^{1,2 *}$, YUQIN PAN ${ }^{2 *}$, BANGSHUN HE ${ }^{2}$, QIWEN DENG ${ }^{2}$, RUI LI ${ }^{1,2}$, YEQIONG XU ${ }^{2}$,

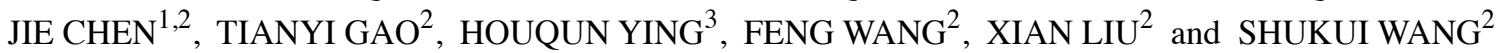 \\ ${ }^{1}$ Department of Life Sciences, Nanjing Normal University; ${ }^{2}$ Central Laboratory, Nanjing First Hospital, Nanjing Medical \\ University, Nanjing, Jiangsu 210006; ${ }^{3}$ Medical College, Southeast University, Nanjing, Jiangsu 210009, P.R. China
}

Received November 7, 2014; Accepted December 29, 2014

DOI: 10.3892/ijo.2015.2852

\begin{abstract}
The recombinant oncolytic adenovirus is a novel anticancer agent to replicate selectively in colon cancer cell lines. Loss of imprinting (LOI) of insulin-like growth factor $2(I G F 2)$ gene is an epigenetic abnormality phenomenon. We utilized the $I G F 2$ LOI in gene therapy for the malignant tumor cell lines. We investigated the tumoricidal effects of IGF2 LOI on four cell lines by oncolytic adenovirus, and constructed novel adenovirus vectors Ad312-E1A and Ad312-EGFP. The expression of E1A was monitored by real-time PCR and western blot analysis. The viability and apoptosis of colorectal cells infected with Ad312-E1A were tested by CCK- 8 and flow cytometry. In addition, we established a colorectal cancer model in nude mice. The results showed that HCT-8 and HT-29 with IGF2 LOI were infected with Ad312-EGFP and then produced the EGFP. Nevertheless, SW480 and GES-1, which were IGF2 MOI, did not produce the EGFP. The Ad312-E1A obviously reduced the cell viability and induced apoptosis in HCT- 8 and HT-29 in vitro, and successfully suppressed tumor growth in HT-29 xenografts in nude mice. In summary, the conditionally replicative adenovirus with loss of IGF2 imprinting system has a positive effect on gene therapy.
\end{abstract}

\section{Introduction}

Colorectal cancer (CRC) is one of the most common malignant tumors of the digestive tract, and the third most malignant tumor in 2013 (1). According to the statistics, the incidence of annual new cases worldwide reached one million

Correspondence to: Professor Shukui Wang, Central Laboratory, Nanjing First Hospital, Nanjing Medical University, 68 Changle Road, Nanjing, Jiangsu 210006, P.R. China

E-mail: shukwang@163.com

"Contributed equally

Key words: IGF2, imprinting system, oncolytic adenovirus, colorectal cancer, gene therapy and nearly half of the deaths, and the occurrence of CRC was reported to occur in a dominantly inherited pattern (2). The traditional treatment of CRC is generally drugs, surgery and chemotherapy, but the effects of these methods remain unsatisfactory. By contrast, the progress of molecular biology has explained that several genes are involved in the carcinogenesis and development of CRC. Hence, efforts should be focused on taking advantage of the genomic imprinting systems to targeted therapy for cancers mediated by oncolytic adenovirus vector.

Insulin like growth factor 2 (IGF2) is located on chromosome $11 \mathrm{p} 15.5$ and expressed predominantly from the paternal allele. IGF2 gene is closely linked to the $H 19$ gene, both IGF 2 and $H 19$ reciprocally regulated imprinted genes and shared enhancers, cis-acting regulatory elements such as the imprinting control region (ICR) (3). Most imprinted genes form clusters or imprinting domains. The ICRs are similar to differentially methylated regions (DMRs). The IGF2 imprinted gene has a momentous function in cell growth, proliferation, differentiation, transformation, apoptosis and the growth and development of embryos, placental formation and metabolism (4) and regulated by enhancers, DMRs, promoter and the transcriptional regulator CCCTC-binding factor (CTCF) (5). Importantly, we successfully came into a new stage to use targeted therapy for malignant tumor based on the loss of IGF2 system (6).

One of the most critical issues of gene therapy is choosing an effective vehicle to deliver therapeutic genes safely and efficiently into target cells. Early region $1 \mathrm{~A}(E 1 A)$ is the first viral gene expressed after human adenovirus (HAdV) infection (7). ElA can influence the cell cycle and prevent apoptosis, making sure viral replication effectively. The adenovirus as a vector for gene therapy has been rapidly developed because of the simple structure, wide host range, high infection rate, easy cultivation and purification (8). Ad5 is commonly used as a gene transfer vector and oncolytic virotherapy using adenoviruses has potential value for therapeutic benefits in malignant mesothelioma. As oncolytic adenovirus particularly replicates and proliferates in tumor cells, the antioncogenes may have an obvious increase in replication, and its expression level may improve hundreds 
Table I. The primers of H19-promoter, E1A and $\beta$-actin.

\begin{tabular}{ll} 
Target gene & \multicolumn{1}{c}{ Primers } \\
\hline H19-promoter & Forward: 5'-AAGTCGACCACCGTTCTATGAAGGGCTTCAGCA-3' \\
& Reverse: 5'-AGAAGCTTGCCCGGGCTTTTTCTAACTG-3' \\
E1A & Forward: 5'-CCCGGATCCGGGCCCTATGAGACATATTATCT-3' \\
& Reverse: 5'-CGCGTCGACCGCAATCACAGGTTTACACCTTA-3' \\
& Sense: 5'-CTGGAACGGTGAAGGTGACA-3' \\
& Antisense: 5'-AAGGGACTTCCTGTAACAACGCA-3'
\end{tabular}

of times to achieve the purpose of killing the tumor cells. The recombinant adenovirus vectors were firstly used as gene therapy in $1985(9,10)$. Now, the recombinant adenoviruses provide a common system for both gene expression studies and therapeutic applications (8).

In the aforementioned studies, the $D T$ - $A$ gene, which was constructed by a recombinant replication adenovirus carrying the $I G F 2$ imprinting system, was specially expressed in the tumor cells (6). In the present study, we further evaluated the efficacy of gene therapy for CRC by constructing the conditional replication-competent adenovirus to provide a novel therapeutic strategy.

\section{Materials and methods}

Ethics statement and nude mice. The present study was carried out in strict accordance with the Guide for the Care and Use of Laboratory Animals of the US National Institutes of Health, and the study protocol was approved by the Committee on the Ethics of Animal Experiments of Nanjing Medical University (SYXK2009-0015). All surgeries were performed under sodium pentobarbital anesthesia, and suffering was minimized as much as possible. Female athymic nude mice at 4 weeks old were obtained from the Experimental Animal Center of University of Yangzhou, Yangzhou, China. The animals were housed in SPF-free facilities with 12-h light-dark cycles and standard pellet feed and water ad libitum.

Cell lines and culture conditions. Human embryonic kidney 293 cells (HEK293) were obtained from the American Type Culture Collection (ATTCC, Manassas, VA, USA). Human colorectal carcinoma cell lines (HCT-8, HT-29 and SW480) and human gastric epithelial cells (GES-1) were purchased by the Shanghai Cell Collection, Chinese Academy of Sciences (Shanghai, China). All the cell lines except SW480 were cultivated in Dulbecco's modified Eagle's medium (DMEM; Gibco-BRL, Grand Island, NY, USA) with $10 \%$ fetal bovine serum (FBS; Gibco) and SW480 cell line was maintained in RPMI-1640 (Invitrogen, Carlsbad, CA, USA) with 10\% FBS. All the cells were incubated under humidified conditions of $95 \%$ air and $5 \% \mathrm{CO}_{2}$ at $37^{\circ} \mathrm{C}$.

Plasmid construction and adenovirus packaging. In the present study, the original adenovirus shuttle plasmid (pDC-312) was used. The mouse $H 19$ enhancer exon 1 (258 bp) and exon 2
(360 bp) and DMR exon 1-2 (429 bp), exon 3 (207 bp) and exon 4 (156 bp) were amplified by PCR from the mouse genomic DNA, respectively, and then the two fragments were linked to a single fragment by PCR. The DMD was cloned downstream of the enhancer by the restriction endonuclease EcoRI and NheI. The mouse H19 promoter (302 bp) was amplified by PCR from mouse genomic DNA with the primers shown in Table I. We used restriction endonuclease SalI/HindIII to clone downstream of the enhancer-DMD. The human adenovirus E1A segment (1013 bp) was amplified by PCR from a TOPK plasmid, which was benevolently provided by Dr Ji-Fan Hu (Stanford University Medical School, Stanford, CA, USA) and the primers are indicated in Table I. The enhanced green fluorescent protein $(E G F P)$ reporter gene from the pEGFP-C1 vector (Clontech Laboratories Inc., Mountain View, CA, USA) and the E1A gene were inserted downstream of $H 19$ promoter by using restriction endonuclease $\mathrm{Bam} \mathrm{HI}$ and HindIII to construct pDC312-enhancer-DMD-H19-EGFP and pDC312-enhancer-DMD-H19-E1A, respectively, which were confirmed by DNA sequencing. The adenovirus Ad312-E1A was constructed by homologous recombination techniques utilizing pDC312-enhancer-DMD-H19-E1A and the adenovirus packaging plasmid PBHGLOX1, 3CRE.

The plasmid carrying E1A gene and the adenovirus vector Ad5 were transfected into HEK293 with liposome Lipofectamine $^{\mathrm{TM}} 2000$ (Invitrogen-Life Technologies, Carlsbad, CA, USA). The Ad312-EGFP is a standard replication deficient adenovirus and constructed by cotransfection of the adenovirus shuttle vector covering EGFP with a deleted E1A/B adenoviral backbone vector. The culture solution was changed after 4-6 h and the cytopathic effect (CPE) was continuously observed through the transfection. The CPE was observed after $\sim 10$ days and the abnormal cells and supernatant were collected, frozen and thawed at $-80^{\circ} \mathrm{C} / 37^{\circ} \mathrm{C}$ three times and centrifuged at 2,500 rpm for $15 \mathrm{~min}$. The supernatant was Ad-E1A and Ad-EGFP. The adenoviruses were plaque purified and propagated in HEK293, then by a $\mathrm{CsCl}$ gradient according to standard techniques purified again. Functional particle titers of all adenoviruses were identified using a plaque assay in HEK293. The explicit control adenovirus (H101) was benevolently supplied by Dr Sheng-Fang Ge (Shanghai Jiao Tong University School of Medicine, Shanghai, China).

Virus infection. The four cell lines were seeded in 96-well plates at a density of 1,000/well for the Cell Counting kit- 8 
(CCK-8; Dojindo Laboratories, Kumamoto, Japan) assay and in 6-well plates at a density of 10\% blot analysis and flow cytometric analysis. The cells were incubated with various concentrations of H101, AdpDC312-E1A and AdpDC312-EGFP with serum-free DMEM at $37^{\circ} \mathrm{C}$ for $60 \mathrm{~min}$. After the incubation period, a normal growth medium replaced the serum-free DMEM with the viruses. The infected cells were expected to continue to be cultured at $37^{\circ} \mathrm{C}$ for further assays.

The EGFP expression analysis in the constructed plasmids. Four kinds of cells (HCT-8, HT-29, SW480 and GES-1) were infected with adenoviral vectors (Ad-EGFP, $10 \mathrm{PFU} / \mathrm{cell}$ ). EGFP expression was examined at $48 \mathrm{~h}$ after infection using a fluorescence inversion microscope system (excitation 450-490 nm type 108; Nikon).

The E1A expression analysis in virus infected cells by real-time PCR. E1A mRNA expression was determined by real-time PCR (RT-PCR; Applied Biosystems, Waltham, MA, USA). Four types of cells (HCT-8, HT-29, SW480 and GES-1) were, respectively, infected with Ad312-E1A (10 plaques forming units/cell). Total RNA was extracted using TRIzol (Invitrogen-Life Technologies) followed by the manufacturer's instructions. The first strand cDNA synthesis was performed in a whole volume of $25 \mu \mathrm{l}: 2 \mu \mathrm{g}$ RNA, $0.5 \mu \mathrm{g}$ up-primer and down-primer, 200 units of M-MLV reverse transcriptase. The cDNA was then amplified in $50 \mu 1$ reaction volumes containing $0.4 \mu \mathrm{mol} / 1$ of up-primer and down-primer as well as 1.25 U Taq DNA polymers (Takara, Dalian, China) with the conditions of pre-denaturation at $94^{\circ} \mathrm{C}$ for $5 \mathrm{~min}$, subsequently, by 35 cycles of $94^{\circ} \mathrm{C}$ for $40 \mathrm{sec}, 60^{\circ} \mathrm{C}$ for $40 \mathrm{sec}$ and $72^{\circ} \mathrm{C}$ for $1 \mathrm{~min}$ and a final extension of $72^{\circ} \mathrm{C}$ for $7 \mathrm{~min}$. The PCR products with 1013 bp were electrophoresed on a 1\% agarose gel with ethidium bromide before visualizing under UV light.

The E1A expression analysis by western blot analysis. Western blot analysis was performed to evaluate the E1A protein level. The HCT-8, HT-29, SW480 and GES-1 cells were washed three times with ice-cold PBS, and the cells were suspended in lysis buffer. Cell lysates were collected through 12,000 rpm, 5 min at $4^{\circ} \mathrm{C}$ and then separated by $12 \%$ sodium dodecyl sulfate polyacrylamide gel electrophoresis (SDS-PAGE) and transferred onto polyvinylidene difluoride (PVDF). Then blocked with 5\% non-fat dry milk in TBST buffer overnight at $4^{\circ} \mathrm{C}$, with mouse anti-E1A antibodies $(1: 1,000)$ and rabbit anti-human $\beta$-actin antibodies (1:500) incubated at $24^{\circ} \mathrm{C}$ for $2 \mathrm{~h}$, and washed by shaking with TBST solution. The proteins were visualized by ECL (Wuhan Boster Biological Technology, Ltd., Wuhan, China). Western blot analyses were performed at least three times.

Cytotoxic effect of E1A by CCK-8 assay. CCK-8 assay was based on the ability of viable cells using a Cell Counting kit-8 (CCK-8; Dojindo Laboratories). The cells were seeded in 96-well plates at a density of 1,000 cells/wells and were infected with recombinant adenoviral vectors (10 PFU/cell) the next day. Following incubation for $72 \mathrm{~h}$, the cells were assayed with CCK-8 reagents by measuring absorbances at $450 \mathrm{~nm}$ with a microplate reader (Bio-Rad Laboratories,
Richmond, CA, USA) to cell growth and viability. All samples were assayed in quadruplicate and the experiments were repeated three times.

Quantative evaluation of apoptosis assay by flow cytometry. The apoptosis assay was performed by flow cytometry after double staining with Annexin V fluorescein isothiocyanate (FITC) apoptosis detection kit (Nanjing Keygen Biotech, Co., Ltd., Nanjing, China) to discriminate early apoptosis (single Annexin V-positive) and double Annexin V/propidium iodidepositive (PI) differentiated necrotic cells. The 6-well dishes were inoculated with $1 \times 10^{6}$ cells/well and then infected with 10 PFU/cell of Ad312-E1A, Ad312-EGFP and H101. Cell apoptosis was analyzed at $72 \mathrm{~h}$ after infection.

Treatment of tumor-bearing nude mice with Ad312-E1A. Tumor xenografts were established by oxter injection of $5 \times 10^{7}$ HT-29 cells into the 4-week-old female athymic nude mice (the Experimental Animal Center of University of Yangzhou, Yangzhou, China). The tumor volume was measured by the formula: Volume $=1 / 2 \mathrm{x}\left(\right.$ length $\mathrm{x}$ width ${ }^{2}$ ). When tumors had grown to $100 \mathrm{~mm}^{3}$, the xenografted mice were randomly divided into four groups of twelve mice in each group. The Ad312-E1A, Ad312-EGFP, H101 received intratumoral injections of $10^{9} \mathrm{PFU}$ every other day. The tumor was calculated by vernier calipers every two days. All the mice were euthanized at 1 month post-inoculation. The mice were euthanized by cervical dislocation at a predetermined interval of observation. The harvested tumors were fixed in $40 \%$ buffered formalin, sectioned at 5-7 $\mathrm{mm}$ and stained with H\&E.

Immunohistochemistry analysis. The tumors were stored in $10 \%$ formalin and embedded in paraffin for staining, and then incubated at $4^{\circ} \mathrm{C}$ overnight with the mouse anti-human E1A antibody (1:50 dilution; Abcam, Boston, MA, USA) to detect E1A protein expression. The sections were rinsed in PBS-T (0.05\% Triton X-100 in PBS) with a goat anti-mouse secondary antibody (1:500 dilution) incubation for $60 \mathrm{~min}$ at room temperature and then incubated with streptavidinhorseradish peroxidase (BD Biosciences, San Jose, CA, USA), diaminobenzidine substrate to form the colorimetric reaction. The positive cells were calculated in six random fields at 400 magnifications with a light microscope. Only distinct staining cells were counted and the positivity rate was utilized to grade the expression levels.

TUNEL assay. Apoptosis of the tumor cells was detected by the terminal deoxynucleotidyl transferase (TdT)-mediated dUTP nick-end labeling (TUNEL) and performed with In Situ Cell Death Detection kit (Roche, Mannheim, Germany) with the manufacturer's direction. The tumors were filled with $10 \%$ formaldehyde and paraffin-embedded sections were prepared to stain apoptotic cells. The number of TUNEL-positive cells was calculated in six random fields at 400 magnification with a light microscope, and then the apoptosis index for each field was calculated as the percent of TUNEL-positive cells relative to the total.

Statistical analysis. IBM SPSS Statistics software version 20.0 (IBM, Armonk, NY, USA) was used to analyze the data. 
Table II. Genomic imprinting of IGF2 and analysis of $p 53$ mutation.

\begin{tabular}{llcc}
\hline Cell line & \multicolumn{1}{c}{ Source of cell } & $p 53$ status & IGF2 imprinting \\
\hline HT-29 & Colon cancer & Mutation & LOI \\
HCT-8 & Colon cancer & Wild & LOI \\
SW480 & Colon cancer & Wide & MOI \\
GES-1 & Human gastric epithelial cell & Wide & MOI \\
\hline
\end{tabular}

LOI, loss of $I G F 2$ imprinting; MOI, maintenance of $I G F 2$ imprinting.

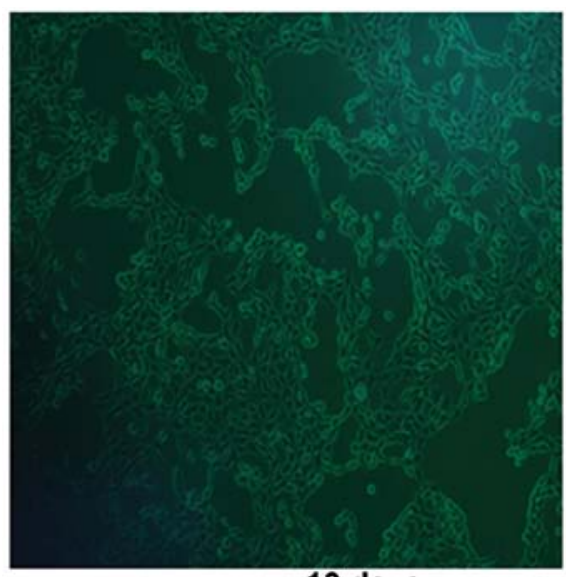

10 days

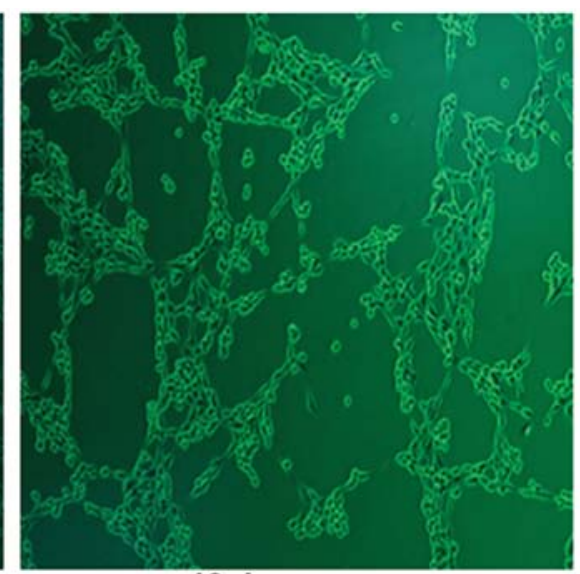

13 days

Figure 1. CPE are shown in HEK293 cells infected with Ad312-E1A. CPE observed at 10 days and 13 days after infected with Ad312-E1A.

Every assay was performed at least three times. All experimental data were expressed as the mean \pm standard deviation and assessed by the Student's t-tests and the one-way ANOVA. The results of in vivo survival experiments were assessed by GraphPad Prism 5 (GraphPad software, San Diego, CA, USA). $\mathrm{P}<0.05$ was considered to be statistically significant.

\section{Results}

Construction and characterization of the oncolytic adenovirus Ad312-E1A. We constructed a conditional replication-competent adenovirus to gene therapy for cancer cells on loss of IGF2 imprinting and p53 mutations as shown in Table II, and cells infected with H101 served as a positive control. Enhancer-DMD fragment (1376 bp) was verified through the restriction endonuclease $\mathrm{SalI}$ and $\mathrm{XbaI}$ from the recombinant plasmids pDC312-enhancer-DMD. The H19 fragment (302 bp) was confirmed through the restriction endonuclease HindIII and SalI from the recombinant plasmid pDC312enhancer-DMD-H19. In addition, E1A (1013 bp) and EGFP (718 bp) fragment were, respectively, verified through the restriction endonuclease BamHI and HindIII from both pDC312-enhancer-DMD-H19-E1A and pDC312-enhancerDMD-H19-EGFP, which were transfected into HEK293 cells, respectively. We observed CPE in HEK293 cells (Fig. 1) after infection with Ad312-E1A at day 10 and day 13. The successful construction of oncolytic adenovirus Ad312-E1A and Ad312-EGFP was confirmed and regulated by the IGF2 imprinting systems, and the Ad312-E1A expressed efficiently and replicated selectively in HCT-8 and HT-29 (IGF2 LOI), except for SW480 and GES-1 (IGF2 MOI). Targeted therapy for CRC was mediated-by oncolytic adenovirus vector, which was based on the IGF2 LOI genomic imprinting systems.

EGFP protein expression in different cells. An EGFP reporter gene was utilized to examine the applicability of the expression system. After infection of the four cell types with Ad312-EGFP (10 PFU/cell) for 48 h, EGFP gene expression was found in LOI cells (HCT-8 and HT-29), but negative or only weakly positive EGFP was observed in MOI cells (SW480 and GES-1) for maintained normal IGF2 imprinting system (Fig. 2). Hence, the virus gene therapy system only expressed the reporter gene in the tumor cells with IGF2 LOI.

E1A $m R N A$ transcript and protein expression. We tested E1A mRNA and protein expression in HCT-8, HT-29, SW480 and GES-1 cells which were, respectively, infected with Ad312-E1A and H101 (10 PFU/cell). The expression of E1A mRNA and protein were determined by RT-PCR and western blot analysis $48 \mathrm{~h}$ after infection, respectively. As shown in Fig. 3, in the Ad312-E1A group, E1A mRNA and protein were expressed in LOI cells (HCT-8 and HT-29), not in MOI cells (SW480) or normal cell (GES-1). The p53 mutant cells (HT-29) infected with H101 lead to high E1A expression in mRNA and protein level, which was hardly expressed in $\mathrm{p} 53$ wild-type cells (HCT-8, SW480 and GES-1), indicating that H101 expressed only in cell lines with p53 mutant. 


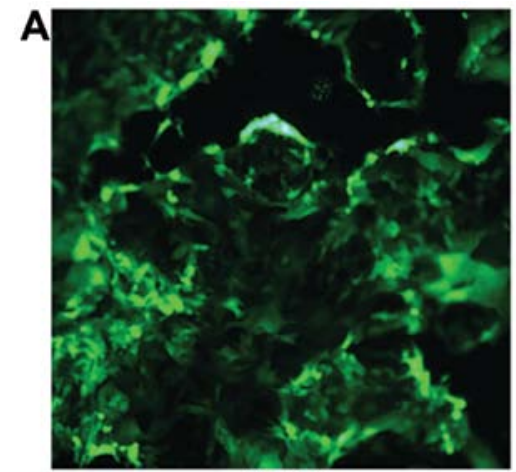

HCT-8

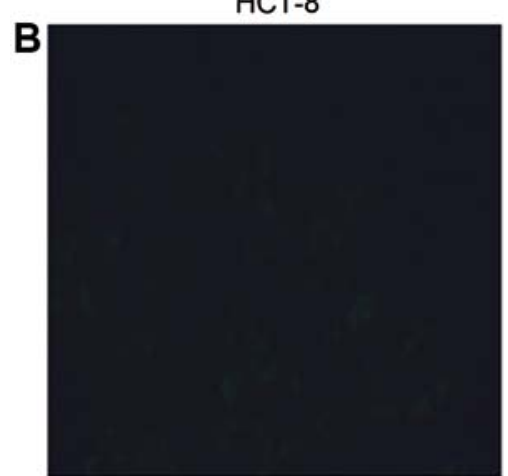

SW480

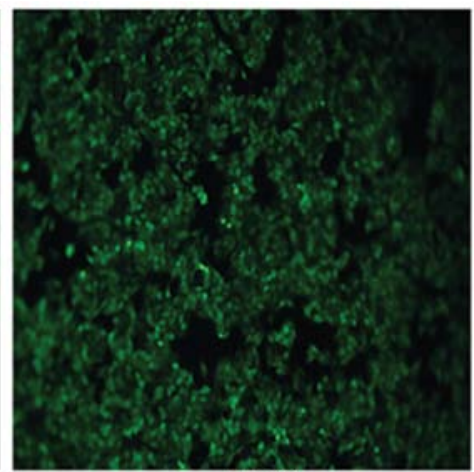

HT-29

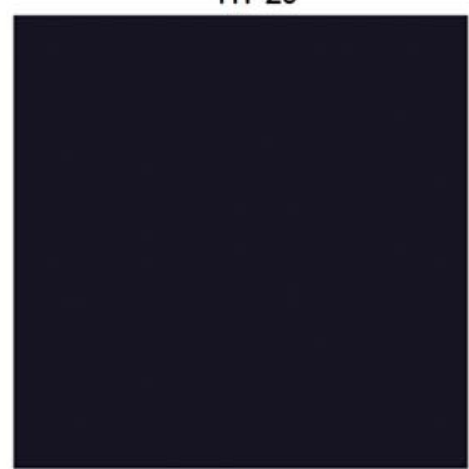

GES-1

Figure 2. EGFP expression in the four cell lines infected with Ad312-EGFP. (A) Infection with Ad312-EGFP (10 PFU/cell) for $48 \mathrm{~h}$ induced expression of EGFP in HT-29 and HCT-8. (B) Negative expression of EGFP in the MOI cell lines. Microscope images showed no EGFP expression in GES-1 and only very weak EGFP expression in SW480 cell lines with Ad312-EGFP (10 PFU/cell).

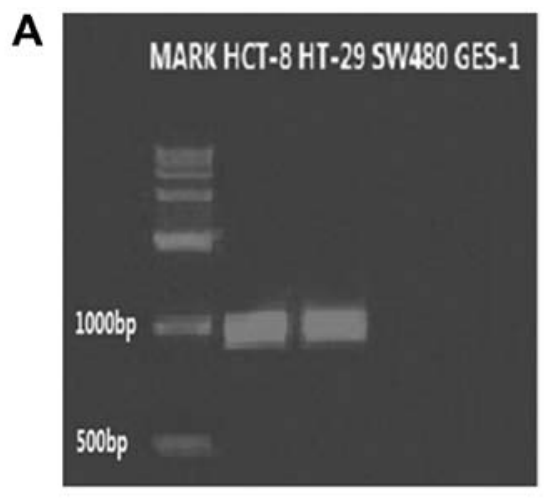

B

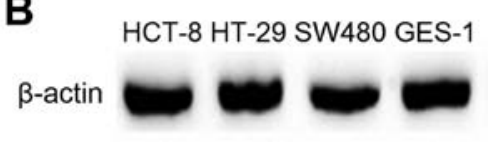

E1A

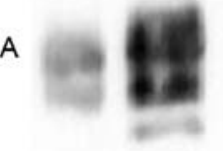

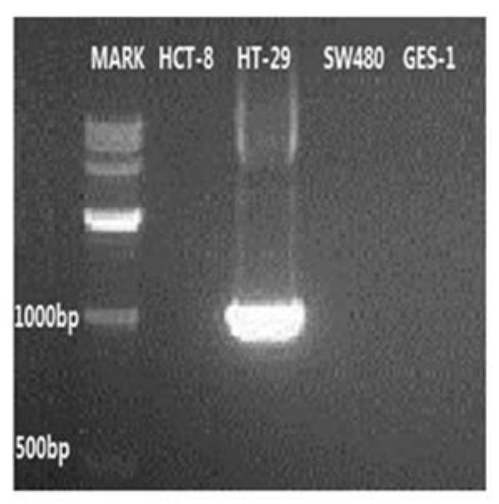

\section{HCT-8 HT-29 SW480 GES-1 \\ $\beta$-actin}

E1A

Figure 3. Both E1A mRNA and protein expression in four cell lines infected with Ad312-E1A or H101 at $48 \mathrm{~h}$. (A) E1A mRNA was expressed in HT-29 and HCT-8 in Ad312-E1A group, and only expressed in HT-29 in the H101 group. (B) E1A protein was expressed in HT-29 and HCT-8 in Ad312-E1A group, and then only expressed in HT-29 in the H101 groups.

Growth inhibition and cytotoxicity of different cell lines by Ad312-E1A infection. The cytotoxic effects of adenoviral vectors expressing E1A were observed in four cell types. The groups were treated with oncolytic adenovirus (10 PFU/cell) Ad312 EGFP, Ad312-E1A, H101 and PBS at $72 \mathrm{~h}$, their viability was assessed by CCK-8 assay (shown in Fig. 4A). 

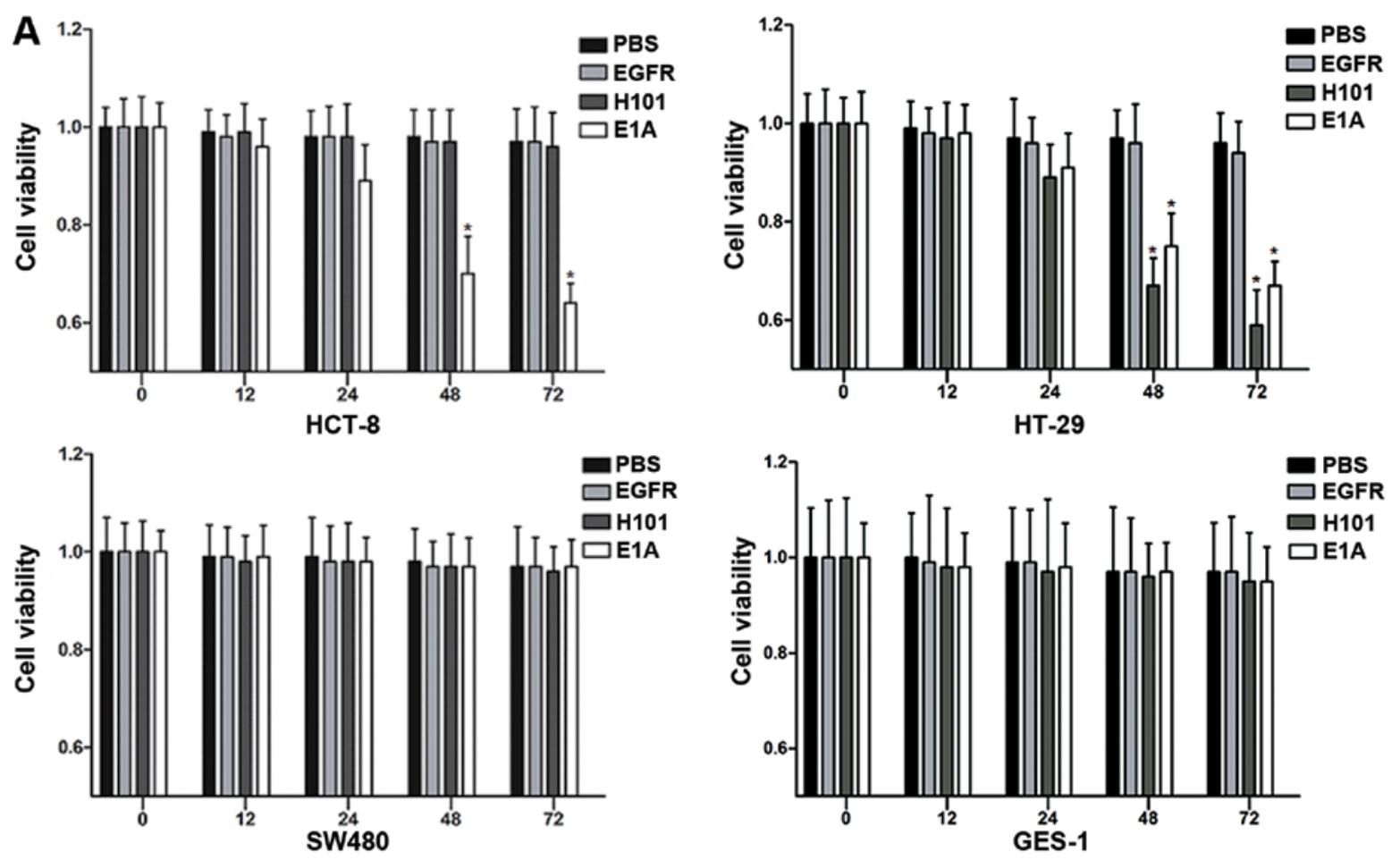

B

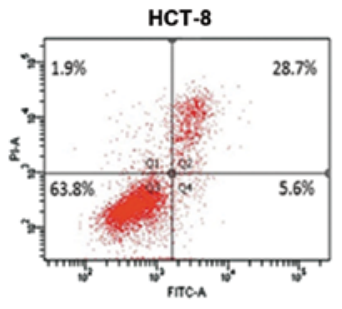

SW480

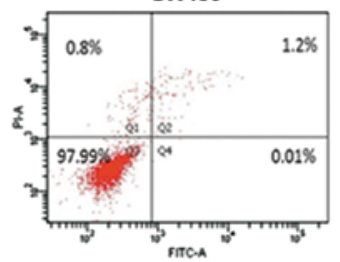

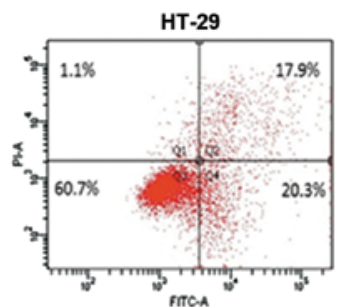

GES-1

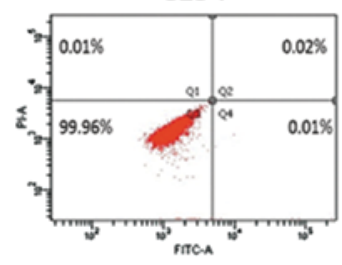

C

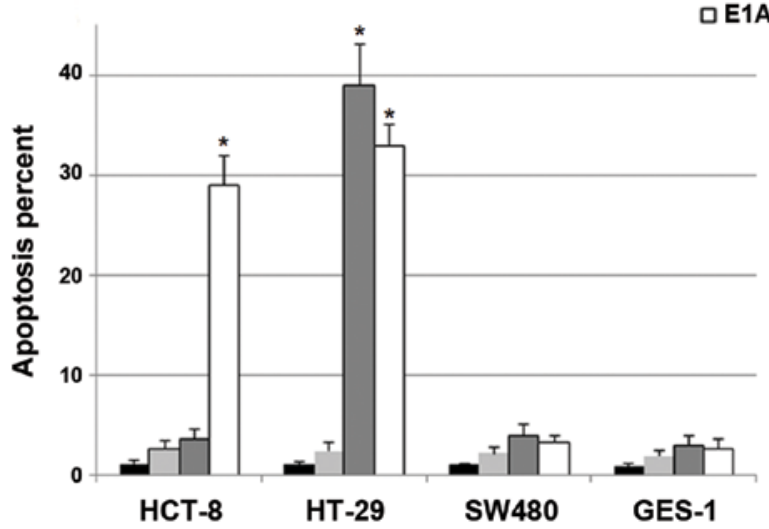

Figure 4. Effect of Ad312-E1A in vitro. Three graphs are representative of different experiments. (A) Cell viability was determined by the CCK-8 assays at 0 , 12, 24, 48 and $72 \mathrm{~h}$ after infection with PBS, Ad312-EGFP, H101 and Ad312-E1A (10 PFU/cell). The cell viability in SW480 and GES-1 cell lines infected with Ad312-E1A show no difference compared with control groups ( $\mathrm{P}>0.05)$. The cell viability in HT-29 and HCT-8 infected with H101 and Ad312-E1A shows a significant decline compared with the PBS and EGFP groups at 48 and $72 \mathrm{~h}\left({ }^{*} \mathrm{P}<0.05\right)$. (B) Apoptosis was tested by using the flow cytometric analysis at $72 \mathrm{~h}$ after infection with Ad312-E1A. (C) The apoptosis was tested in four cell lines infected with Ad312-E1A. The apoptosis rate in LOI cell lines (HT-29 and HCT-8) were obviously increased compared to control groups $\left({ }^{\#} \mathrm{P}<0.01,{ }^{*} \mathrm{P}<0.05\right)$. The data are representative of three different experiments.

The result of CCK-8 assay revealed that the cell viability of the LOI cells (HCT-8 and HT-29) infected with Ad312-E1A was significantly reduced when compared with the MOI cells (SW480 and GES-1) $(\mathrm{P}<0.05)$, which still had higher cell viability. In the same way, the viability of p53 mutant cell line (HT-29) infected with oncolytic adenovirus H101 was also significantly decreased in contrast to the $\mathrm{p} 53$ wild cell lines (HCT-8, SW480 and GES-1) $(\mathrm{P}<0.05)$, which had obviously stronger cell viability.

Cell apoptosis induced by the Ad312-E1A. Apoptosis in the four cell types was calculated using flow cytometry $72 \mathrm{~h}$ after Ad312-E1A infection. To evaluate the cytopathic effect of adenoviral infection on the cells, apoptosis of cells infected with Ad312-EGFP was measured as the negative control (Fig. 4B). The resutls indicated that the apoptosis rate in LOI cell lines (HCT-8 and HT-29) infected with Ad312-E1A (10 PFU/cell) was significantly higher than that in the control group $(\mathrm{P}<0.05)$. However, there was no significant difference of apoptosis ratio between MOI cells (SW480 and GES-1) infected with Ad312-E1A (10 PFU/cell) and the negative control group $(\mathrm{P}>0.05)$. To evaluate the cytopathic effect of H101 and Ad312-E1A on the p53 mutant cells (HT-29), similar experimental procedure was applied, and the results showed no obvious difference between the two groups $(\mathrm{P}>0.05)$, as shown in Fig. 4C. 

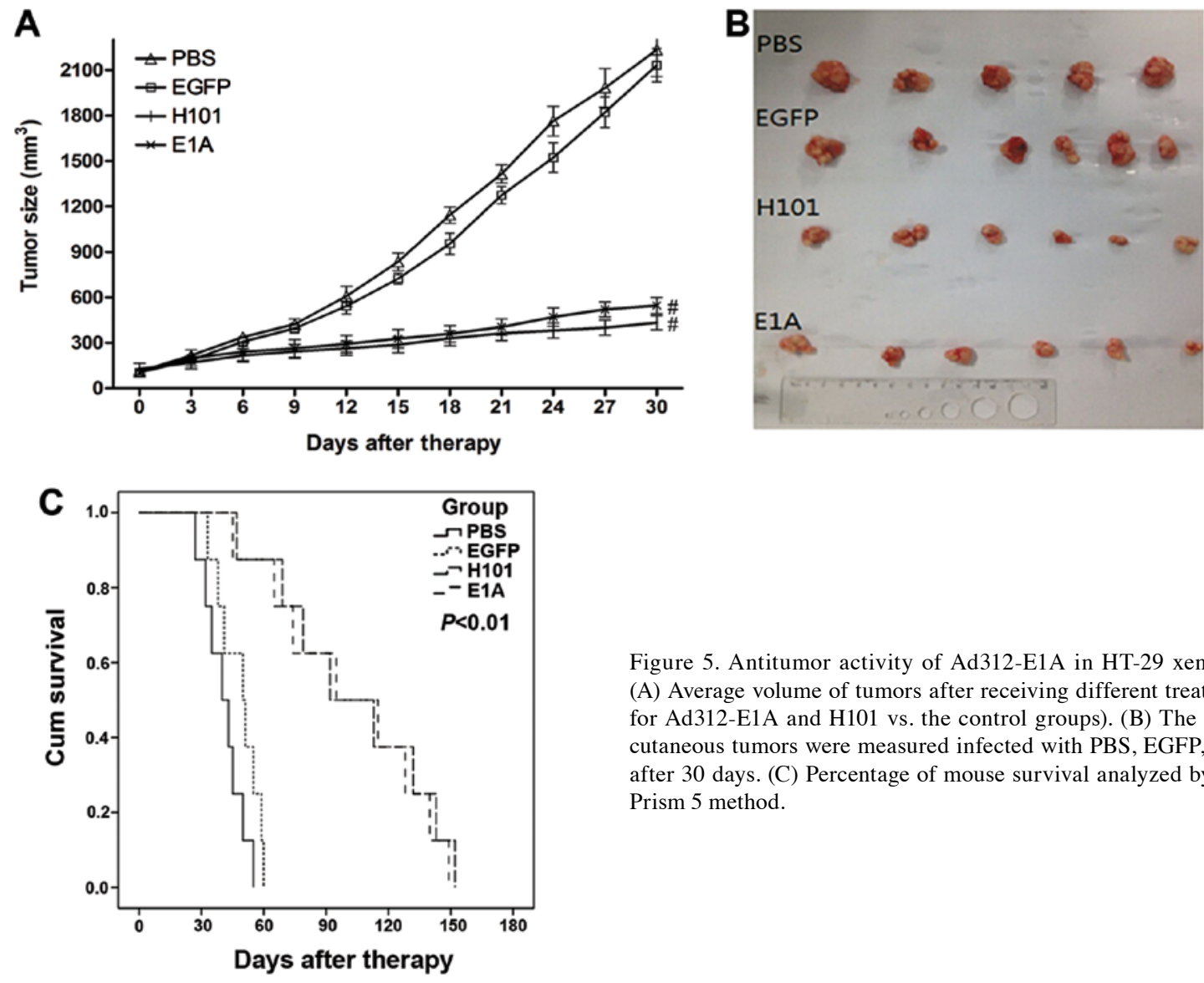

Figure 5. Antitumor activity of Ad312-E1A in HT-29 xenograft models (A) Average volume of tumors after receiving different treatments $\left({ }^{*} \mathrm{P}<0.01\right.$ for Ad312-E1A and H101 vs. the control groups). (B) The volume of subcutaneous tumors were measured infected with PBS, EGFP, H101 and E1A after 30 days. (C) Percentage of mouse survival analyzed by the GraphPad Prism 5 method.

In vivo antitumor effect of the Ad312-E1A. The antitumor effect of the recombinant adenoviral was tested in nude mice transplanted with HT-29 cells. We measured tumor volume once every three days for 30 days after injecting Ad312-E1A, H101, Ad312-EGFP and PBS ( $n=12$ per group), and the average volume of the tumors were 432, 498, 2132 and $2233 \mathrm{~mm}^{3}$, as shown in Fig. 5A. After 30 days of injection, the tumor tissues were harvested, as shown in Fig. 5B. Although no significant difference existed between infected with Ad312-E1A and H101 groups, a significant antitumor efficacy was shown in these two groups compared with that in PBS and Ad312-EGFP groups $(\mathrm{P}<0.01)$. In addition, the average survival time of the four groups treated with Ad312-E1A, H101, Ad312-EGFP and PBS were 149, 152, 60 and 55 days, as shown in Fig. 5C. In short, the above results showed that the survival time of group with infection of Ad312-EGFP was not significantly prolonged compared with the group injected with PBS ( $P>0.05)$, and that the survival time of the group injected with the Ad312-E1A and H101 was obviously prolonged compared with PBS group $(\mathrm{P}<0.05)$.

Immunohistology by TUNEL assay. To detect apoptosis in tumors from mice injected with Ad312-E1A, Ad312-EGFP, H101 and PBS, TUNEL assay was applied after the tumor tissue obtained, the number of apoptotic bodies in tumor tissue after staining with hematoxylin and eosin (H\&E) was higher in the two groups with Ad312-E1A and H101 infection than in the group treated with Ad312-EGFP and PBS (shown in Fig. 6A and B). Furthermore, the expression of E1A protein was confirmed in tumor tissue from mice injected with Ad312-E1A or H101, as shown in Fig. 6C. In addition, the apoptosis index, represented by the percentage of TUNEL-positive cells, in the group of H101 $(0.63 \pm 0.04)$ and E1A $(0.59 \pm 0.05)$ was higher than that in group of PBS $(0.19 \pm 0.06)$, EGFP $(0.27 \pm 0.02)$, separately $(\mathrm{P}<0.01)$. Moreover, the expression rate of E1A in tumour tissue of the four groups was $0.009 \pm 0.001$ in PBS group, $0.01 \pm 0.002$ in Ad312-EGFP group, $0.43 \pm 0.06$ in H101 group and 0.48 \pm 0.08 in Ad312-E1A group, respectively, as shown in Fig. 6D. In conclusion, cell apoptotic indexes of tumour tissue from xenograft mice in groups treated with both Ad312-E1A and H101 were obviously higher than in the groups treated with the PBS and EGFP, separately $(\mathrm{P}<0.05)$, and the apoptotic indexes of xenograft tumors treated with Ad312-E1A had no difference compared with the H101 group $(\mathrm{P}>0.05)$.

\section{Discussion}

Genomic imprinting is involved in hominoid epigenetic regulation. In recent years, $I G F 2$, characterized by genomic imprinting, is an important autocrine/paracrine growth factor in tumors for its mitogenic and antiapoptotic functions (11). However, it is also reported that the imprinting status of the $I G F 2$ gene is closely associated with somatic overgrowth and embryonal tumors related to several different malignancies in human (12-18). Hence, in the present study, the novel replication-selective adenovirus Ad312-E1A and Ad312-EGFP were constructed based on loss of the $I G F 2$ imprinting, and the data 
A PBS

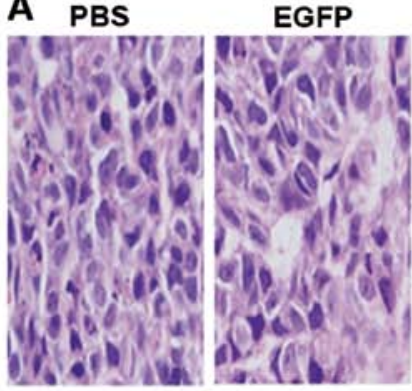

\section{PBS}

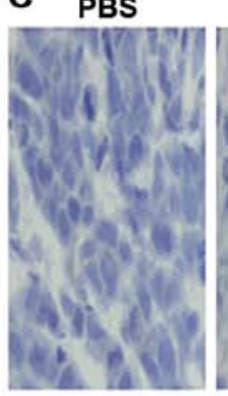

EGFP

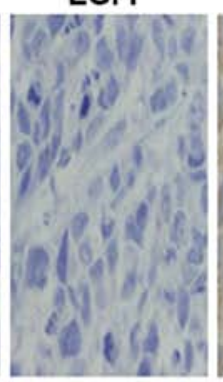

H101

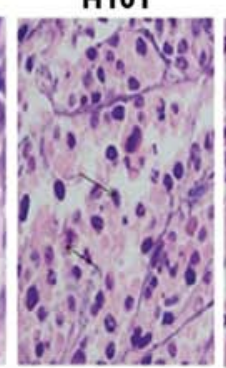

H101

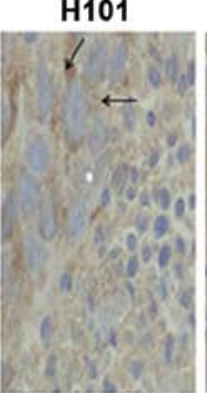

E1A

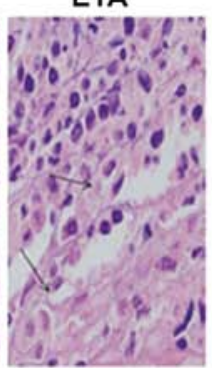

B

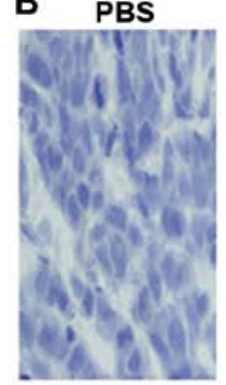

EGFP

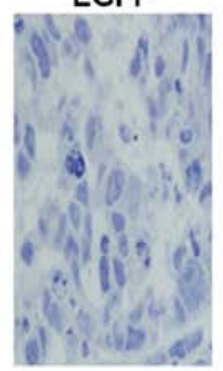

H101

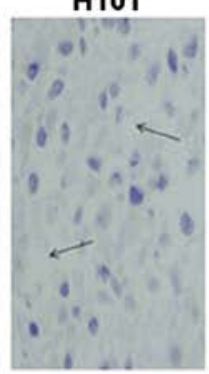

E1A

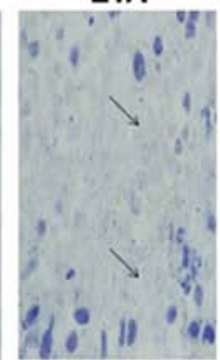

E1A D
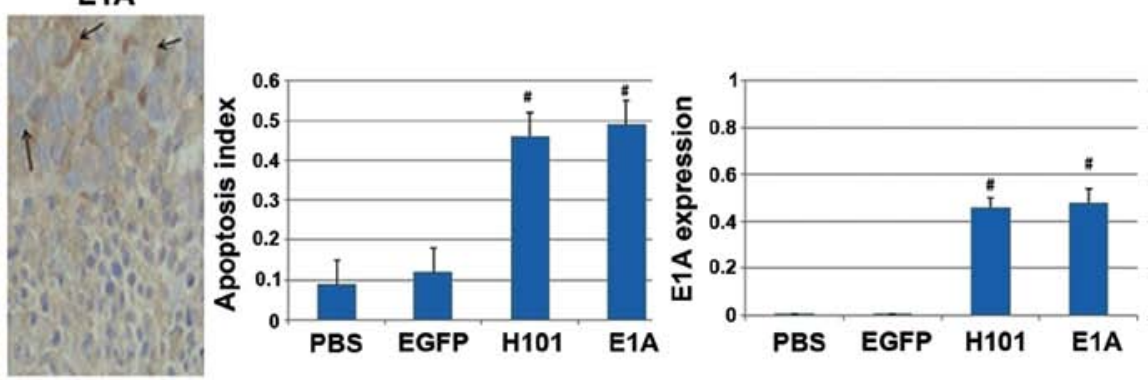

Figure 6. In vivo, enhanced E1 A expression and apoptosis through Ad312-E1A treatment. (A) Histopathological responses in tumors on days 30 after treatment (H\&E staining; original magnification, $\mathrm{x} 400$ ). (B) The tumor cell apoptosis was measured by a TUNEL assay after treatment. (C) Immunohistochemical staining in tumors to detect the E1A protein expression. The E1A protein expression is shown in four different groups (original magnification, $\mathrm{x} 400$ ). (D) Analysis of the E1A apoptosis percentage and expression rate. Both E1A apoptosis and expression show a significant increase compared with the control groups $(" \mathrm{P}<0.01)$.

based on the oncolytic adenovirus Ad312-E1A displayed a meaningfully effect on suppressing tumor growth both in vitro and in vivo, indicating that replication-selective adenoviruses carrying the IGF2 imprinting system can be used as a new type of antitumor agent with a high therapeutic potential of clinical treatment.

For $I G F 2$ gene, in general, only paternal alleles expressed and maternal alleles closed, which is known as the maintenance of imprinting (MOI); however, the imprinted $I G F 2$ had abnormal expression by the reactivation of suppressed maternal IGF2 allele unregulation $(19,20)$, which is known as loss of imprinting (LOI), triggered by the abnormal binding of insulator CTCF to H19 ICR (21-23), which caused by the impaired function of CTCF or the hypomethylation status of DMR in the ICR region.

Overexpression of $I G F 2$ accelerated the growth of tumor cells causing tumorigenesis by influencing a bioactive peptide to promote mitosis function (24).

The IGF2 LOI was detected in tumor tissues and cell lines by several methods according to the published reports. In the Reeve and Feinberg laboratories, the imprinted $I G F 2$, transcribed exclusively from paternal allele while maternal allele remains silent, could be identified by the restriction fragment length polymorphism (RFLP) method applied for the detection of the an ApaI digestion single nucleoside polymorphism (SNP) in exon 9 of IGF2 $(25,26)$. The ribonuclease protection assays (RPA) method, a sensitive technique for detection and quantification of RNA expression, has been reported to detect the imprinting status of the $I G F 2$ gene $(27,28)$. Previous studies based on chromatin conformation capture (3C) method or chromatin immunoprecipitation (ChIP) have confirmed the imprinting status of $I G F 2$ in tumor cell lines $(I G F 2 \mathrm{LOI}$ :
HCT-8, HRT18, HT-29, HCT15, T84, Caco-2 and SW1222; IGF2 MOI: SW1116, SW480, HCT116 and LIM1215) (29-31). In the present study, the IGF2 LOI cell lines (HCT-8 and HT-29) and IGF2 MOI cell lines (SW480 and GES-1) were utilized for gene therapy.

$I G F 2$ LOI as a hallmark of various human neoplasms has been widely investigated in somatic overgrowth and embryonal tumors related to breast (12), prostate (13), lung cancer (14), renal cell carcinoma (15), esophageal $(16,17)$, ovarian cancer (18) and Wilms' tumor (26). Moreover, Baba et al (31) demonstrated an association between IGF2 DMR hypomethylation and clinical prognosis, and expounded its potential role as a prognostic biomarker in more than 1,000 patients of CRC. In addition, upregulation expression of $I G F 2$ has been detected in CRC $(32,33)$, indicating $I G F 2$ LOI may serve as a potential biomarker in diagnosis of CRC (34).

IGF2 LOI involved in the pathology of cancers has trigged research interest. The present study carried out gene therapy based on oncolytic virus H101 and Ad312-E1A for CRC in vivo and in vitro. $\mathrm{H} 101$, an adenovirus with the E1B-55KD and pretrial E3 deleted, is the oncolytic adenovirus with the most extensive investigation in that it could selectively replicate in tumor cells rather than in normal cells, resulting in specific tumor cytolysis, and was first applied in clinical treatment of squamous cell carcinoma of head and neck in China (35). Ad312-E1A, a tumoricidal gene, is constructed by the replication-defective adenovirus Ad312, which has been verified as a promising vector system for the treatment of malignant diseases by influencing the cell cycle, preventing apoptosis, and making sure of viral replication (36). In the present study, Ad312-E1A was observed to be 
safe in xenografts in mice, in vivo. Regardless of the fact that, the reconstruction virus combined with IGF2 LOI and Ad312-E1A or H101 was confirmed with antitumor efficacy, the gene therapy based on them should be addressed with some caution. Firstly, the oncolytic adenovirus Ad312-E1A, which carried loss of $I G F 2$ imprinting system, has a positive effect on the cells with $I G F 2$ LOI. Moreover, the oncolytic virus $\mathrm{H} 101$ was expressed in p53 mutant cells with IGF2 LOI (HT-29). Secondly, the reconstruction virus discussed in the study involved in a small number of $\mathrm{CRC}$, and further research is required using other types of cancer on the loss of IGF2 imprinting, such as Wilms' tumor, leukemia, osteosarcoma, leiomyosarcoma, breast cancer, lung cancer and hepatoma.

In conclusion, in the present study, the conditionally replicative adenovirus Ad312-E1A, which carried loss of $I G F 2$ imprinting system, has a positive effect on CRC cell lines with IGF2 LOI, indicating that the gene therapy based on Ad312-E1A and IGF2 LOI could act as a novel strategy for CRC therapy.

\section{Acknowledgements}

The present study was supported by grants from The National Nature Science Foundation of China (no. 81172141 and 81200401), the Nanjing Science and Technology Committee Project (no. 201108025), the Nanjing Medical Technology Development Project (no. ZKX11025), the Nanjing Health Young Talent Project, Jiangsu Provincial Key Medical Talents to S.K.W., and the Nanjing Medical Science and Technique Development Foundation to Y.Q.P. (no. QRX11255 and YKK13107) and B.S.H. (no. QRX11254).

\section{References}

1. Siegel R, Naishadham D and Jemal A: Cancer statistics, 2013. CA Cancer J Clin 63: 11-30, 2013.

2. Houlston RS, Collins A, Slack J and Morton NE: Dominant genes for colorectal cancer are not rare. Ann Hum Genet 56: 99-103, 1992.

3. Fu VX, Dobosy JR, Desotelle JA, et al: Aging and cancer-related loss of insulin-like growth factor 2 imprinting in the mouse and human prostate. Cancer Res 68: 6797-6802, 2008.

4. Engstrom W, Shokrai A, Otte K, et al: Transcriptional regulation and biological significance of the insulin like growth factor II gene. Cell Prolif 31: 173-189, 1998.

5. Engel N, Thorvaldsen JL and Bartolomei MS: CTCF binding sites promote transcription initiation and prevent DNA methylation on the maternal allele at the imprinted H19/Igf2 locus. Hum Mol Genet 15: 2945-2954, 2006.

6. Pan Y, He B, Li T, et al: Targeted tumor gene therapy based on loss of IGF2 imprinting. Cancer Biol Ther 10: 290-298, 2010.

7. Nevins JR, Ginsberg HS, Blanchard JM, Wilson MC and Darnell JE Jr: Regulation of the primary expression of the early adenovirus transcription units. J Virol 32: 727-733, 1979.

8. Breyer B, Jiang W, Cheng H, et al: Adenoviral vector-mediated gene transfer for human gene therapy. Curr Gene Ther 1: 149-162, 2001.

9. Yamada M, Lewis JA and Grodzicker T: Overproduction of the protein product of a nonselected foreign gene carried by an adenovirus vector. Proc Natl Acad Sci USA 82: 3567-3571, 1985.

10. Ballay A, Levrero M, Buendia MA, Tiollais P and Perricaudet M In vitro and in vivo synthesis of the hepatitis B virus surface antigen and of the receptor for polymerized human serum albumin from recombinant human adenoviruses. EMBO J 4 $3861-3865,1985$.

11. Pollak MN, Schernhammer ES and Hankinson SE: Insulin-like growth factors and neoplasia. Nat Rev Cancer 4: 505-518, 2004.
12. Ito Y, Koessler T, Ibrahim AE, et al: Somatically acquired hypomethylation of IGF2 in breast and colorectal cancer. Hum Mol Genet 17: 2633-2643, 2008.

13. Kaneda A and Feinberg AP: Loss of imprinting of IGF2: a common epigenetic modifier of intestinal tumor risk. Cancer Res 65: 11236-11240, 2005.

14. Suzuki H, Ueda R and Takahashi T: Altered imprinting in lung cancer. Nat Genet 6: 332-333, 1994.

15. Oda $H$, Kume $H$, Shimizu $Y$, Inoue $T$ and Ishikawa $T$ : Loss of imprinting of igf 2 in renal-cell carcinomas. Int $\mathrm{J}$ Cancer 75 : 343-346, 1998.

16. Mori M, Inoue H, Shiraishi T, et al: Relaxation of insulin-like growth factor 2 gene imprinting in esophageal cancer. Int $\mathrm{J}$ Cancer 68: 441-446, 1996.

17. Hibi K, Nakamura H, Hirai A, et al: Loss of H19 imprinting in esophageal cancer. Cancer Res 56: 480-482, 1996.

18. Yun K, Fukumoto M and Jinno Y: Monoallelic expression of the insulin-like growth factor-2 gene in ovarian cancer. Am J Pathol 148: 1081-1087, 1996.

19. Li T, Hu JF, Qiu X, et al: CTCF regulates allelic expression of Igf2 by orchestrating a promoter-polycomb repressive complex 2 intrachromosomal loop. Mol Cell Biol 28: 6473-6482, 2008.

20. Rakha EA, Pinder SE, Paish CE and Ellis IO: Expression of the transcription factor CTCF in invasive breast cancer: a candidate gene located at 16q22.1. Br J Cancer 91: 1591-1596, 2004.

21. Yang Y, Hu JF, Ulaner GA, et al: Epigenetic regulation of Igf2/ H19 imprinting at CTCF insulator binding sites. J Cell Biochem 90: 1038-1055, 2003.

22. Paradowska A, Fenic I, Konrad L, et al: Aberrant epigenetic modifications in the CTCF binding domain of the IGF2/H19 gene in prostate cancer compared with benign prostate hyperplasia. Int J Oncol 35: 87-96, 2009.

23. Szabo PE, Tang SH, Silva FJ, Tsark WM and Mann JR: Role of CTCF binding sites in the Igf $2 / \mathrm{H} 19$ imprinting control region. Mol Cell Biol 24: 4791-4800, 2004.

24. Liu M, Roth A, Yu M, et al: The IGF2 intronic miR-483 selectively enhances transcription from IGF2 fetal promoters and enhances tumorigenesis. Genes Dev 27: 2543-2548, 2013.

25. Rainier S, Johnson LA, Dobry CJ, Ping AJ, Grundy PE and Feinberg AP: Relaxation of imprinted genes in human cancer. Nature 362: 747-749, 1993.

26. Ogawa O, Eccles MR, Szeto J, et al: Relaxation of insulin-like growth factor II gene imprinting implicated in Wilms' tumour. Nature 362: 749-751, 1993.

27. Ekstrom TJ, Cui H, Li X and Ohlsson R: Promoter-specific IGF2 imprinting status and its plasticity during human liver development. Development 121: 309-316, 1995.

28. Ohlsson R, Nystrom A, Pfeifer-Ohlsson S, et al: IGF2 is parentally imprinted during human embryogenesis and in the Beckwith-Wiedemann syndrome. Nat Genet 4: 94-97, 1993.

29. Nakagawa H, Chadwick RB, Peltomaki P, Plass C, Nakamura Y and de La Chapelle A: Loss of imprinting of the insulin-like growth factor II gene occurs by biallelic methylation in a core region of $\mathrm{H} 19$-associated CTCF-binding sites in colorectal cancer. Proc Natl Acad Sci USA 98: 591-596, 2001.

30. Cui H: Loss of imprinting of IGF2 as an epigenetic marker for the risk of human cancer. Dis Markers 23: 105-112, 2007.

31. Baba Y, Nosho K, Shima K, et al: Hypomethylation of the IGF2 DMR in colorectal tumors, detected by bisulfite pyrosequencing, is associated with poor prognosis. Gastroenterology 139: 1855-1864, 2010.

32. Tricoli JV, Rall LB, Karakousis CP, et al: Enhanced levels of insulin-like growth factor messenger RNA in human colon carcinomas and liposarcomas. Cancer Res 46: 6169-6173, 1986.

33. Lambert S, Vivario J, Boniver J and Gol-Winkler R: Abnormal expression and structural modification of the insulin-like growth-factor-II gene in human colorectal tumors. Int J Cancer 46: 405-410, 1990

34. Cui H, Cruz-Correa M, Giardiello FM, et al: Loss of IGF2 imprinting: a potential marker of colorectal cancer risk. Science 299: 1753-1755, 2003.

35. Garber K: China approves world's first oncolytic virus therapy for cancer treatment. J Natl Cancer Inst 98: 298-300, 2006.

36. Holm PS, Lage H, Bergmann S, et al: Multidrug-resistant cancer cells facilitate E1-independent adenoviral replication: impact for cancer gene therapy. Cancer Res 64: 322-328, 2004. 